\title{
Communication, Technology, and the Planetary Creature
}

\author{
Giuseppe O. Longo
}

Dipartimento di Elettrotecnica Elettronica Informatica, University of Trieste, longo@units.it

\begin{abstract}
Man and technology are inseparable: man produces technology, but the latter contributes to the continuous transformation of man. Today biological evolution based on random genetic mutations is largely exceeded by cultural (more specifically, technological) evolution, which is much faster due to the presence of Lamarckian inheritance mechanisms (imitation, learning and the like). This has two important consequences: 1) the formation of Homo technologicus, a symbiotic creature where biology meets technology intimately and is subject to a continuous transformation; and 2) the formation of a sort of Planetary Creature that originates from the interconnection of the individual man-machine symbionts and is heralded by the Internet and by the communication activities occurring in the Internet, in particular those taking place in the so-called social networks.

The Planetary Creature is a single world-wide structure where important communication and cognitive processes occur, developing into a sort of connective intelligence that tends to absorb individual intelligences. This encroaching process can produce resistance and dissent as well as suffering, but can also enhance individual abilities. Actually, the growing efficiency and the decreasing costs of the communication mediated by technology offer unprecedented opportunities for augmenting knowledge and creativity and for eliciting novel forms of intellectual activity. On the other hand there might be negative consequences such as addiction to computers and virtuality, delegation of activities and abilities to machines, vulnerability of complex systems, undue control on individuals and economic exploitation. Some important consequences of these developments are examined concerning the body, the time and space categories, and in particular the identity concept.
\end{abstract}

Keywords: Internet, Homo Technologicus, Planetary Creature, Connective Intelligence, Social Networks

The following text is the keynote address of Guiseppe O. Longo at the 9th International Conference on Sociocybernetics, organised by the Research Committee 51 of the International Sociological Association (ISA) in Urbino, 29 June - 5 July 2009. The theme of the conference was: "Modernity 2.0" - Emerging Social Media Technologies and Their Impacts. Longo presents here the perspective of engineering science towards sociological issues. He has published several books on that topic, so far in Italian language only. Though some of his statements in the text don't seem well-founded to sociological readers or may be deemed highly speculative, we decided to make his ideas accessible to the non-ltalian world. We think they are worth to be. Wolfgang Hofkirchner

\section{The Communication Explosion}

We are witnessing a rapid and huge increase of communicative exchanges caused and mediated by the information and communication technology (ICT). The main causes of such communication outburst are

- the increase of technical efficiency

- the dramatic decrease of costs

- the weakening of the traditional filters that hindered the spreading of information (religion, school, family, the society at large). Such filters were supported by the cost of communication, by the widespread illiteracy and by the slowness of communication exchanges.

The ICT has made communication increasingly rapid and cheap and has contributed to the formation of what is called Information Society. A particular aspect of that technology is the increasing integration and convergence of different technological areas:

- convergence between telecommunications and computers

- digitalization of all sources and channels of information

- development of multimedia interactive services 
- concentration of a host of functions in pocket-small devices

The judgments of the technological progress vary from utterly positive, such as the information society offers unprecedented opportunities for increasing knowledge and creativity, and for strengthening democracy and participation to totally negative, such as: dependency on computers, delegation of activities and abilities to machines, vulnerability of complex systems, undue control on individuals by a sort of Orwellian Big Brother, addiction to virtual reality, anxiety from faster and faster interactions, new forms of illiteracy, threatens to democracy, loss of the sense of communication and possibly of life.

What such a variety of contrasting judgments suggests is that we are facing a dramatic revolution, largely rooted in the interaction between technology and society. It is becoming evident that the true vocation of computers is not number crunching or data processing as much as interactive connection among individuals. Computers are more and more seen as nodes of the communication network that is rapidly extending all over the planet.

Communication is a complex phenomenon, hence it can (and has to) be described at different levels and from several different points of view, none of which can give a thorough description: as a result, the phenomenon of communication gives the impression of ambiguity. In other words, both positive and negative assessments can be justified by different observers with very convincing arguments.

No matter whether we judge positively or negatively the fact that everybody can communicate his or her own thoughts, sensations and impressions, however significant or insignificant, to a large number of individuals at a very low cost, we should not forget that under the surface of the communication phenomena there is a powerful economic system aiming at gaining money and power through

cunning market policies and advertising strategies.

\section{The Advent of Homo Technologicus}

When we consider the problem of the relations between humans and technology, we often assume tacitly that man and technology are distinct and separate entities. Moreover, we assume that technology evolves very quickly, whereas human evolution is very slow or even still. My premises here are different: on one hand, the distinction between man and technology is not sharp, since technology has always had a big role in shaping the intimate nature of man and, on the other hand, technological evolution, more generally cultural evolution, has gradually taken the place of biological evolution and has become a sort of continuation of it.

These two evolutions have become strictly intertwined and have formed a "bio-cultural" or "bio-technological" evolution which has set the stage for the appearance of a new (pseudo)species, Homo technologicus, a symbiotic creature where biology meets technology intimately. Homo technologicus is not simply "Homo sapiens plus technology", but rather "Homo sapiens transformed by technology": it is a new evolutionary unit, which undergoes a new kind of evolution in a new environment.

The bio-technological evolution is ruled by a mixture of Darwinian and Lamarckian mechanisms. We know that the mechanism of inheritance of acquired characters proposed by Jean-Baptiste Lamarck to explain biological evolution does not work, because it would soon lead a species into an evolutional deadend. But it does work in cultural evolution, where imitation and learning are powerful and quick mechanisms that shortcut the long and slow selection process proposed by Charles Darwin to explain biological evolution. Due to Lamarckian mechanisms, technological (and in general cultural) evolution is rapid: quick adaptations to novelties tend to root immediately into the deep structure of society. The rapidity of this evolution, however, also causes its products to be fragile.

If the scenario I am sketching here is credible, and if Homo technologicus will really emerge from history and evolution, what are to be the consequences? Rather than in the continuity aspects of the shift from biological evolution to bio-technological evolution, I am interested in the discontinuities and in the mismatch between the organic and the artificial components of the symbiont. No doubt, 
the two evolutions are somewhat heterogeneous and, consequently, the two components of the man-machine hybrid are heterogeneous. This mismatch can cause certain kinds of suffering which would add to those due to our organic nature, although technology contributes to relieving some "conventional" forms of pain.

The deepest human characteristics, those associated with emotions, communication, expression, the atavic inheritance linked to the body and rooted in the most ancient layers of evolution, which played a fundamental role in the survival and development of our species, would not disappear at once only because technology would insert in our bodies and brains its nanometric prosthetic devices. And in the contact area, in the infinitesimal interface between "us" and "our" protheses, serious rejection processes could show up. Even today, when Homo technologicus is still in an early stage of development, we can observe troubles and difficulties due to the mismatch and incompatibility between man and machine. As an evidence of this, remark that a great deal of research efforts is being devoted to the construction of "user friendly" machines, which should create an anaesthetic zone in which the artificial components are allowed to creep. In other words, the ancient body components try to oppose to the encroachment of the newer mind constructions and we purposefully try to weaken this resistance.

The consequences of this course of action are difficult to predict, but all this could be a source of problems. In the technology cage that we are building around ourselves as a tight suit, some of our skills will be as useless as prehistoric relics, but will nevertheless continue to demand to be put to use or will take as phantom limbs. Other skills will obviously be enhanced: technology will operate a sort of selective filtering on our persons (complex units of mind and body).

In Homo Technologicus the original biological base is increasingly invaded, hybridized and transformed by artificial devices: thus Homo technologicus is gradually becoming a cyborg. And such evolution is more and more rapid, since technological innovation is accelerating due to a positive feedback: the presence of technology facilitates the introduction of more technology. Positive feedback loops cause acceleration in processes, but also a dangerous instability that can even lead to breakdown or runaway phenomena.

\section{Potential Knowledge and Actual Knowledge}

Thanks to a series of communication instruments, represented by spoken language, writing, printing, all the way down to the ICT tools, among which the Internet is the most recent, human beings have increased the amount and velocity of exchanged messages, by which they can influence one another's thought processes. So the brains of different individuals united by language can be harnessed together in ways, which would be impossible without language. A whole group on individuals can be integrated and can start to behave as a single thinking entity.

With the development of the ICT, the range over which this integration could take place has extended gradually to the whole planet: in such a "global village" the amount of exchanged messages and of shared knowledge, stored in larger and larger memories, increases steadily. As a consequence, thanks to the ease of access, the potential mass of knowledge available to each individual augments constantly. On the other hand the amount of actual knowledge, i. e. knowledge that an individual can pour into his personal memory and can process remains more or less constant in time. There is an increasing gap between the collective (potential) knowledge and the individual (actual) knowledge.

The communicative unification produced by language and its supporting technologies extends into a cognitive unification: thought and knowledge achieve a cooperative dimension, so that some scholars have coined the expression "collective intelligence", meaning that the social communication activity supports a social cognitive activity at a superindividual level.

This is partly a metaphor, but there is no doubt that some intelligent activities of great importance, e. g. some significant scientific or industrial enterprises, are only made possible by a vast collection of cooperating minds connected by language and communication. No 
single mind could ever perform experimental or theoretical activities whose complexity exceeds a given level.

In other words, the communicational overheating supported by ICTs brings about the formation of a sort of nervous system of the planet, or Planetary Creature, a web-like structure whose meshes connect human beings and computers to perform cooperative cognitive activities. Between such collective intelligence and the individual intelligences there are quantitative as well as qualitative differences. Quantitatively, the collective intelligence is more and more powerful, whereas individual intelligences have been more or less constant for thousands of years. On the other hand, from a qualitative point of view, each individual intelligence is rooted in a particular body and is accompanied by emotions, feelings and by an individual consciousness that belongs to the holder of the intelligence. The Planetary Creature, or collective mind, does not possess, at least for the moment, emotions or a consciousness.

It is interesting to note that the development of the Creature is not guided from outside, rather it is self-organized and emerges from chaos.

The widening gap between the individual and the collective cognitive abilities demands an increasing recourse to a sort of technological delegation: to process the increasing amount of information we have to resort to machines, which in turn allows an increase in the information amount that can be processed, in a positive feedback loop. Such delegation process might extend to the decision process, and this would make the attribution of responsibility equivocal and problematic.

\section{The Bio-Technological Evolution}

As I said, differently from biological inheritance, cultural inheritance is not limited to parent-offspring transmission: it works also among individuals of the same generation by imitation and learning processes. Hence cultural novelties can spread very quickly through society and among societies, thus cultural evolution is much more rapid than biological evolution, although its products are more fragile and volatile. It should be empha- sized again that the two evolutions are not independent: on one hand cultural evolution is conditioned by biological constraints, on the other hand every cultural novelty can play a role in biological evolution, especially - but not only - when it concerns the process of reproduction: new fertilization techniques, social mating rules, retention and support of handicapped children, genetic manipulations, etc.

The increasing speed of biocultural evolution and the resulting acceleration in the formation rate of the successive symbionts are significant phenomena, as they are the main cause of the mismatches and sufferings that are always present in hybridation phenomena and their products.

It seems that today the velocity of the manmachine hybridation overcomes our ability to adjust the two components to each other, and that brings forth suffering and pain. In particular this is true of mind machines, that are the most subtle and pervasive devices and are responsible for the most remarkable transformations in humans.

In the 20th century along with the traditional machines for processing energy and matter, we started to construct machines that process information. Also, miniaturization allows implantation of micro- and nano-devices directly in the body or brain, enhancing traditional capabilities or even creating new, unprecedented ones. Such hybridation processes bring forth a continuous wave of emergent properties that foreshadow the so-called posthuman stage of evolution, the more so as electronic communication is making worldwide integration of human beings a reality in the form of the Planetary Creature.

In past times the continuous transformation of Homo technologicus and its hybrid nature were not visible, which justified the conception of man as a steady and stable species. Today, however, the rapid development of technology has shown that Homo technologicus undergoes a continuous series of changes. Among such changes, let me single out the consequences of an early exposure of children to computers, playstations and the like. It seems that the brain connections of those children tend to develop in a different way relative to children that are brought up tradi- 
tionally, e. g. reading, writing, and playing with mates. As a consequence, school teachers and children have different brain structures, as teachers were brought up in a more traditional environment, hence teachers and pupils have difficulties in communicating with each other. This might be one of the reasons of the difficulties in which school struggles today.

The phenomena that I have briefly sketched out, i.e.

- the man-machine hybridation leading to Homo technologicus,

- the world-wide integration of humans bringing about a cognitive Planetary Creature,

- the mismatch between biological and artificial components of the symbiont, with consequent sufferings,

- the technological delegation of abilities and responsibilities to machines,

- the emergence of new and unpredictable abilities in Homo technologicus, together with the weakening or disappearance of traditional abilities,

all these and other related phenomena that I will touch upon later, show how deep and strong the influence of technology on society is.

Without lapsing into a sort of technological determinism, I wish to stress the importance of mind machines in shaping social activities, first of all communication, learning, trade and business. It is on the basis of such considerations that the adoption of new technologies should be evaluated.

In cultural evolution the engine of change resides in conscious purpose rather than in random mutations. Conscious purpose, operating through a more and more powerful technology, is a strong catalyzer of social, cultural and even biological transformations. The real source of innovation is probably the still mysterious mechanism of creativity, which we qualify as random for lack of a deeper understanding. But to be implemented, new ideas have to pass the sieves of cultural compatibility, social acceptance and economic feasibility: due to these filters many innovations do not even reach the stage of embryo and die unknown to people.
However, the results achieved by conscious purpose often do not coincide with the planned ones: the outcomes of biocultural evolution can be at variance with the wished outcomes, in spite of all earnest and industrious endeavors. Such a phenomenon originates from the interaction of human plans and designs with the complexity of the real world that limits and constrains the scope, extent and direction of our projects. As a consequence, uncertainty breaks into history, evolution, market. In particular, the relations between society and technology are unpredictable, and this is partly why sociology is an interesting discipline.

Moreover, the uncertainty in the evolution of socio-technology is increased by the fact that many innovations are unfinished and do not have definite boundaries. Designers often construct seeds or embryos of innovations, that later evolve under the effect both of design rules and of random interactions with the environment. Those embryonic innovations intertwine with existing systems to form inextricable tangles. Such is the case of genetically modified crops that come in contact with traditional plants triggering unpredictable developments. Think also of software programs that are launched on the market to be tested and improved by users. Or think of social networks, whose evolution depends on the contributions of thousands or millions of users and, within the technical constraints, can undergo unpredictable developments and twists. This is self-organization at work: coherent phenomena emerging from chaos.

To make a long story short, our ability to act and construct has now surpassed our ability to understand and foresee. In this sense technology has outdone science.

Let me now go back to the Planetary Creature to remark that this cognitive entity represents a further evolutionary stage with respect to Homo sapiens and Homo technologicus, a stage where collective or connective intelligence holds sway over individual intelligences. The whole of mankind seems on the way to become rapidly a virtually single organism, or a single community like a beehive or an ant colony. Social insects exhibit a collective intelligence, and a collective behavior, that depend on the intense and permanent 
communication activity among the units. The same is happening to human beings connected by language and by ICT.

Mankind will continue to evolve, more and more rapidly, but intellectually rather than physically. There is, however, a basic difference between the Planetary Creature and an ant or bee colony: as far as we know, the individual intelligence of social insects is very low, whereas human individuals have high cognitive abilities and also feelings, emotions and consciousness. This raises the question whether humans are willing to give up these characteristics, partly or totally, to merge into the Planetary Creature. They might oppose a diffuse resistance to renouncing their most intimate and personal qualities and experiences, including free will. Moreover, certain ancestral traits, such as competition and aggression, could oppose the formation and development of the Planetary Creature.

On the other hand, many collective or coherent behaviors are to be observed in contemporary society, especially among young people, and this might denote a shift towards cooperation, which could ease collective conducts and actions.

As to the issue of democracy on line, some observers believe that the cheap and easy access offered by the Internet could allow an exercise of democracy based on a direct and immediate knowledge of events. For instance when totalitarian governments obscure or ban newspapers and radio and TV stations, the Internet might be the only source of information for people, thus triggering and supporting popular movements and initiatives. Under normal circumstances, everybody could put any issue to the vote just pushing a button after gathering enough relevant information. Actually, however, this raises many problems:

- who, and on what basis, decides when information is enough?

- who, and on what basis, decides when a piece of information is relevant?

Actually, news are put online by someone, hence they are chosen and filtered depending on overt or hidden interests. Checking the news is difficult and time-consuming, and this would inevitably lead (as in fact has already led) to the creation of mediation organs, as is usual in more traditional media with press agencies. People would resort to these organs rather than to the primary sources of information (i. e. the events themselves), difficult to identify and to check, so democracy would be severely limited. Should one insist on having first-hand news, he would risk spending all his time online, thus renouncing his real life.

As to the general features of the Planetary Creature, it will possess many of the characteristics of an organism, hence it will tend strenuously to preserve and to expand itself at the expenses of an external (real and virtual) space whose entropy is bound to increase beyond any limit. Such entropy might appear under the form of sufferings for some fringes: the poor, the social misfits, the outcasts. Such fringes would develop resentful feelings and frustration, and could represent a menace for the affluent classes.

Another possible hindrance to the development of the Planetary Creature is the limitation of some important concrete resources: space, energy, air and water supply and quality. Also the increasing complexity of mind machines could pose some problems, especially when they will be inserted in the brains of people. All this could give rise to a certain degree of instability, whose consequences are difficult to foresee. As we know, the behavior of complex systems is sensitive to initial conditions, and even a slight modification of such conditions could provoke a large effect in due time (the so-called butterfly effect). Almost all processes and phenomena, traffic, pollution, crime, the use of drugs, distribution of wealth, etc. are complex and partially ungovernable, and any of them could run out of control to cause a disaster on a planetary scale. Actually, globalization makes all perturbations propagate quickly all over the world.

Also, there are informational limitations for the Planetary Creature: the flood of data produced by the communication overheating could paralyze the system due to technical deficiencies or devastating virus epidemics. The demand for complete transparency, which is often put forward, is also likely to cause harm: paradoxically, the shadowless 
world of total access and transparency could be unsafe for communication. It is not an accident that the greatest part of the information exchanges and processes of a society are unknown to the majority of its members or, in the case of an organism, are kept at the level of unawareness. Otherwise the society or the organism would be stuck and could not function properly.

\section{The Evolution of the Planetary Creature: A Scenario}

Assume now that in spite of all obstacles the Planetary Creature develops into a fullfledged organism, absorbing the individual cognitive abilities. That would represent a new evolutionary stage of mankind, on which I wish to speculate briefly.

Since the Planetary Creature is one and only, it has neither companions nor competitors with which to hold a dialogue or to contend. Therefore it would lack one of the most powerful driving force behind change and evolution.

In principle the Planetary Creature might govern its own further evolution following rational criteria and exercising a perfect control over its own progress. But this raises a problem: what would urge the Planetary Creature to evolve? Why should it evolve? In other words: what would be its needs, deficits, or longings? Why should it modify its blissful and rapturous state since no competitor would threaten it and no one would complain about its flaws and limitations? Actually, the Planetary Creature might persist forever in a stationary and unperturbed state, without any advancement.

But this stationary picture might be deceptive: the Planetary Creature would be confronted both with the shifts and drifts of its internal dynamics as well as with external constraints (time, space, entropy, water, food...). Actually the Creature would not dwell in a void or in the virtual space of pure information, rather, due to its cyborganic components (Homines technologici), it would extend into physical reality. Hence the Creature's survival and integrity would depend on physical reality and material resources, and it would have to tackle environmental alterations and pollution, climate changes, energy and water shortage, deterioration of equipment, the turnover and possibly the rebellion of some of its own component cells (man-machine symbionts).

Also, it would have to deal with information entropy and degradation, such as logical paradoxes and viruses that would spring up spontaneously or by deliberate intervention of mutinous subsystems. It is difficult to conceive a Planetary Creature that would last for a long lapse of time without undergoing change and differentiation. Energy dynamics and information drives would lead to perturbations, emergent novelties, phase changes, instabilities, chaos.

We might wonder whether that dynamics could be accompanied by the emergence of a sort of dim, primitive consciousness. Actually there is no reason to believe that the myriad of individual consciousness could or should give rise to a collective consciousness in the same way as individual cognitions give rise to a collective cognition. There can be cognitive activities without consciousness, as it happens in artificial intelligence, hence the Planetary Creature should not necessarily possess a consciousness. In humans consciousness is a product of evolution, therefore it probably has had some survival value, which is questionable for the Planetary Creature.

Also, human beings are semantic entities, i. e. they always (try to) find some sense in what they do, perceive, feel, touch, experience. Humans are complex nodes where the long threads of ethics, aesthetics, experience, memory, projects, impulses, instincts, longings etc. twine and wind and weave to make the fabric of what we call our world. And this fabric is made up of stories: ceaselessly we tell stories or are told stories, articulate monologues, dialogues, fictions, and perform informal theatre pieces. In short we construct a linguistic world, superimposing it to the real world.

Hence the question: could the Planetary Creature be or become a semantic "machine"? Could it tell meaningful stories? Our semantics is deeply rooted in our bodies: could the semantics of the Creature be rooted in its body? And what is that body likely to be? Our body is not only the material structure that contains our organs, including the brain, it is also our personal history, it accumulates our 
experiences, we know the world by means of our bodies: joy, sorrow, pain, hope, love, hatred are in the body as well as in the mind. Actually the distinction between mind and body is artificial. All important events in our lives happen in and through our bodies: birth, love, sex, delivery, disease, well-being and death. Are equivalent or similar events possible in the Creature?

The ineffable coloration of our actions, feelings, hopes, grieves, joys that we call sense is within each of us, and we try to launch a bridge towards the others, a bridge made of communication: words, glances, postures, smiles, tears. Over that bridge our own sense endeavors to meet other people's and establish a contact based on our common specific origin and nature and common individual experiences. But with whom or what would the Planetary Creature try to share its sense, if any? What stories or origin myths would it contrive to justify its existence to itself and to anticipate its future? Are blogs, chats, forums, social networks and the like the primordial seeds of a new kind of narration or simply a blurred and random ground noise?

\section{Social networks}

Now I'll touch briefly on social networks, one of the most interesting phenomena belonging to the vast landscape of communication mediated by technology. Many of my previous considerations can be applied to social networks. However they have some characteristic features and raise some peculiar problems. The main issues are:

$$
\begin{array}{ll}
\text { - } & \text { time } \\
\text { - body } \\
\text { - } \\
\text { - } \text { identity } \\
\text { contents }
\end{array}
$$

As to the contents of the messages, it is extremely variable. The enormous increase in quantity probably goes with an average decrease of quality with respect to the past, when only important messages were exchanged due to the high transmission cost. Today innovative and significant messages go with trivialities, mental gurglings, narcissistic and egotistic trifles ("this morning I had cornflakes at breakfast - oh, great!').

At the same time there is always someone in the huge audience who is interested even in the faintest information ripplings and is willing to reply, possibly triggering a lively dialogue that can expand rapidly and die out rapidly.

The increase of exchanges increases the communicating amount of people and consequently the human thinking mass. Beyond a given threshold, this quantitative increase can lead to qualitative variations of intelligence. In any case, the technological interface tends to wipe out many components of direct communication, such as metamessages, nonverbal messages, tone of voice and the like.

A substantial part of interlocutors, assuming that this term is still valid, never had direct, face-to-face contacts, so people address to a public of unknown individuals, whose identity is vague or identifies with a name, or a pseudonym, or a nickname. This may lead to a sensation of irresponsibility, even impunity, that in turn pushes to counterfeit one's identity, sending messages that could have been sent by a completely different subject. On the other hand, identity is not defined completely by the sender, rather emerges from a cooperative construction in which the receiver is involved too. Different receivers attribute more or less different identities to the same sender. Thus we discover that identity, like information or beauty, is a relative entity, i. e. originates from the interaction between the sender and the receiver. In fact, we do not have a precise and explicit notion of our own identity until we are obliged to specify it, e. g. when we have to submit it to other people (possibly forging it).

We are caught in an interplay of mirror images, we are conditioned by the representation we offer of ourselves to other people, there might be an underlying psychological mechanism connected with mirror neurons, an imitation game that we play with ourselves: you should be careful when you present an identity to other people, because that identity might become your real identity. If you pretend to be honest and fair or mean and base, you might become fair or mean. Moreover, the conventional identity we offer to other people usually persists stable whereas we undergo a 
sequence of transformations. Neurophysiologists have discovered that each of us possesses many personalities, one of which, in turn, holds sway: how do we get away with being considered different from what we feel we are in that moment? Identity could readily become a cliché.

There are other problems concerning identity: in a restricted sense, if 'identity' coincides with a set of personal data that we entrust to the net, identity can be forged, and even stolen, by someone willing to commit illegal deeds. This raises the problem of privacy and abuses, such as the construction of profiles of potential customers of goods or services or the selection of applicants for a given job.

This has to do with the thorny issue of the relation between security and freedom, in the light of possible abuses by authorities or financial groups, companies or other institutions. Many prefer security and are prepared to sacrifice privacy and freedom, at least partly. Others, to oppose abuses and data thefts, might choose to construct paranoid armoured online communities, giving up the traditional, or promised, transparency of the web.

As I said, the messages posted in the social networks are extremely varied. Sometimes they are assertive and self-assured, lacking however rationalization and strong arguments. This is typical of the web culture, and exhibits the mosaic structure of that culture, in which every piece is interesting, but none is really fundamental. Many users only wish to assert their existence and presence, sort of waving to the invisible (or partially visible) audience. It is an ongoing reality show, or web-show, that overflows into the 'real' everyday reality to erase it or at least to blur it.

The consequence of this confusion between real reality and virtual reality is not that virtual reality emerges as real, rather that real reality emerges as virtual: the virtualization of all experiences is one of the many facets of epistemology that technology has brought to the forestage. Such a virtualization exhibits the traits of epistemological constructivism: reality is out there, but we cannot reach it directly and our experience of it depends on our interactions with it: it is the filter of interactions that transforms reality from virtual, or potential, into actual.

Another consequence of this emphasis on virtuality is that some people try to escape into virtual worlds: they create fake identities, more gratifying than those offered to relatives and other close people in everyday life and hide behind those forged identities. E. g. shabby housewives distressed by daily chores or discouraged man afflicted with a thankless job strive sometimes to disappear into the web. Of course this is not possible, but many people spend a lot of their time online. Diving into a social network can be very gratifying and can even have some of the effects of a drug, including addiction phenomena. The addiction syndrome and the corresponding withdrawal symptoms can appear also at the elementary level of e-mail.

As an extreme example of escape from reality into the net, I mention the "hikikomori" phenomenon, well known in Japan, by which many teenagers refuse to live a normal life, with face-to-face relations with their parents and friends and turn to contacts mediated by the net. Their virtual world absorbs them completely and were not for some minimal vital needs, like eating and drinking, they would happily or unhappily disappear into the cyberspace. We can only try to imagine what the experience of time, space, and reality may be for these youngsters.

As the hikikomori phenomenon suggests, spending the majority of one's time in the cyberspace not only modifies the notion, perception and experience of time, but also results in a gradual vanishing of the body, whose importance for our identity and ontology I have already stressed. Semantics, i. e. the sense of the world and of our presence and action in the world, stems from the primary necessity of preserving body's integrity and to satisfy its basic needs. Hence, if the body disappears, our semantics is deeply disturbed. By the way, these phenomena confirm the deep influence that technology exerts on our categories, cognitive abilities and weltanschauung (view of the world).

Even if we ignore such pathological cases, whose consequences on the psycho-physical well-being are devastating, also a moderate (or normal, in the West) use of technology has 
important consequences. The increasing speed of communication brings about compulsion, anxiety, mental overload, restlessness, inattention to other activities. The pressure to react immediately to the stimuli (messages) may hinder careful reflection.

We wish and tend to be connected all the time, lest we be excluded from the big communication game. We have to be always reachable, we have to be at disposal of anybody who might make an offer, a proposal, a suggestion, put a question or make a remark. At the same time this exposes us to a myriad of incoming messages, most of which unwanted, that invariably take our minds off track. It is as we were particles in a fluid subject to a random Brownian motion: communication is fragmented, and so is time, and the fabric of our relations is torn up. Thus, on one hand such communicative perturbations increase our possibilities, on the other hand they destroy our concentration.

Moreover, the expression "free time" has lost its meaning: since the Internet has crossed the thresholds of our houses, it has increasingly absorbed our time and has blurred the distinction between labor time and free time. We are always busy. Life-long learning is one of the signs of this ceaseless slavery. And, no matter how much effort we put in it, we cannot catch up, since we are crushed by the huge, and increasing, disproportion between us and the Internet (i. e. the massive horde of other people). For whom do we work so hard?

Time is really the key resource, not only we parse our activities, perceptions and feelings in time, but we live in time. Time is the only thing that we cannot increase or expand. Time is irreversible, so we have to be careful as how we spend it. Either we live our lives or we observe other people's life. Either we privilege action or we privilege communication or observation. We can do both things, of course, but time is limited, if we devote more time to one thing we subtract it from other things. If we pursue and watch other people's activities we are diverted from our own time, activities and life.

Be wise administrators of your time!

\section{References}

Longo, G. O. (2001). Homo Technologicus. Roma: Meltemi.

Longo, G. O. (2003). I/ simbionte: prove di umanità futura. Roma: Meltemi.

Longo, G. O. (2008). II senso e la narrazione. Milano: Springer Italia.

Marchesini, R. (2002). Post-human. Torino: Bollati Boringhieri.

\section{About the Author}

Giuseppe O. Longo

Giuseppe O. Longo has done research work in information theory and algebraic coding theory. His current interests concern artificial intelligence, human and machine communication and the relationship between man and technology. As a writer he has published three novels and several short stories collections. He is a playwrite and many of his pieces have been staged at various theaters or broadcast by radio. 